\title{
SYSTEMS OF MEROMORPHIC MICRODIFFERENTIAL EQUATIONS
}

\author{
ORLANDO NETO \\ CMAF, Universidade de Lisboa \\ Av. Prof. Gama Pinto, 2, 1699 Lisboa, Portugal \\ E-mail: orlando@ptmat.lmc.fc.ul.pt
}

\begin{abstract}
We introduce the notion of system of meromorphic microdifferential equations. We use it to prove a desingularization theorem for systems of microdifferential equations.

1. Introduction. Let $X$ be a complex manifold. Let $\mathcal{E}_{X}$ be the sheaf of microdifferential operators on $T^{*} X$. Let $Y$ be an hypersurface of $X$. Let $j: T^{*}(X \backslash Y) \rightarrow T^{*} X$ be the open inclusion. Let $\mathcal{E}_{X}^{\prime}$ be the sheaf of sections $P$ of $j_{*} j^{-1} \mathcal{E}_{X}$ such that, given a local generator $f$ of $I_{Y}, f^{k} P \in \mathcal{E}_{X}$, for $k$ large enough. The restriction to the zero section of $\mathcal{E}_{X}^{\prime}$ equals the ring $\mathcal{D}_{X}[Y]$ of differential operators on $X$, meromorphic along $Y$. Nevertheless, the sheaf $\mathcal{E}_{X}^{\prime}$ is not a ring. Actually, if $Y$ equals $\{x=0\}$ then

$$
\partial_{x}^{-1} x^{-1}=\sum_{k \geq 1} k ! x^{-k} \partial_{x}^{-k}
$$

The ring $\mathcal{E}_{X}$ is deeply related with the geometry of $T^{*} X$. In order to localize $\mathcal{E}_{X}$ along a hypersurface of $T^{*} X$, this hypersurface should be "special".

Let $Y$ be a normal crossings divisor of $X$. Let $\pi: T^{*}\langle X / Y\rangle \rightarrow X$ be the fiber bundle with sheaf of sections the locally free $\mathcal{O}_{X}$-module $\Omega_{X}^{1}\langle Y\rangle$ of logarithmic differential forms. We will built in $T^{*}\langle X / Y\rangle$ a sheaf $\mathcal{E}_{X}[Y]$ such that $\mathcal{E}_{X}[Y]$ equals $\mathcal{E}_{X}$ outside $\pi^{-1}(Y)$ and the restriction of $\mathcal{E}_{X}[Y]$ to the zero section equals the $\operatorname{ring} \mathcal{D}_{X}[Y]$. The sheaf $\mathcal{E}_{X}[Y]$ is a microlocalization of the sheaf $\mathcal{D}_{X}[Y]$ in the same sense as the sheaf $\mathcal{E}_{X}$ is a microlocalization of the sheaf $\mathcal{D}_{X}$. We call a coherent $\mathcal{E}_{X}[Y]$-module a system of meromorphic microdifferential equations. We call the $\mathcal{E}_{X}[Y]$-module $\mathcal{E}_{X}[Y] \otimes_{\mathcal{D}_{X}[Y]} \mathcal{N}$ the microlocaliza-

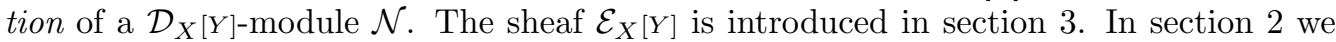
have summarized without proofs the relevant material on logarithmic contact manifolds (see [N1] and [N2]). In section 5 we use the blow ups introduced in section 4 to prove
\end{abstract}

1991 Mathematics Subject Classification: Primary 58G15; Secondary 35A20, 35N15, 35S99. Research partially supported by contract Human Capital and Mobility CHRXCT930082. The paper is in final form and no version of it will be published elsewhere. 
a desingularization theorem for holonomic systems of microdifferential equations in two variables. This result will be used elsewhere to study the local structure of such systems.

2. Logarithmic contact manifolds. Let $X$ be a complex manifold. We will denote by $\mathcal{O}_{X}$ the sheaf of holomorphic functions on $X$. Given a subvariety $Y$ of $X$, we will denote by $I_{Y}$ the ideal of $\mathcal{O}_{X}$ of holomorphic functions that vanish on $Y$. We say that $Y$ is a normal crossings divisor of $X$ if for each $y_{0} \in Y$ there is an open neighbourhood $U$ of $y_{0}$ and a system of local coordinates $\left(x_{1}, \ldots, x_{n}\right)$ on $U$, centered at $y_{0}$, such that

$$
Y \cap U=\left\{x_{1} \ldots x_{\nu}=0\right\} .
$$

If condition (1) holds, we will say that the system of local coordinates $\left(x_{1}, \ldots, x_{n}\right)$ is adapted to the normal crossings divisor $Y$.

We will denote by $\Omega_{X}^{*}$ the complex of sheaves of differential forms with holomorphic coefficients. A differential form $\alpha$ of degree 1 with meromorphic coeficients is said to be a logarithmic differential form with poles along $Y$ if $I_{Y} \alpha \subset \Omega_{X}^{1}, I_{Y} d \alpha \subset \Omega_{X}^{2}$. We will denote by $\Omega_{X}^{1}\langle Y\rangle$ the $\mathcal{O}_{X}$-module of logarithmic differential forms with poles along $Y$. We define $\Omega_{X}^{p}\langle Y\rangle=\wedge^{p} \Omega_{X}^{1}\langle Y\rangle$. Under the assumption (1),

$$
\left.\Omega_{X}^{1}\langle Y\rangle\right|_{U}=\sum_{i=1}^{\nu} \mathcal{O}_{U} \frac{d x_{i}}{x_{i}}+\sum_{i=\nu+1}^{n} \mathcal{O}_{U} d x_{i}
$$

If $\omega=\sum_{i=1}^{\nu} a_{i} d x_{i} / x_{i}+\sum_{i=\nu+1}^{n} a_{i} d x_{i}$ is a section of $\Omega_{X}^{1}\langle Y\rangle$ on $U$, we define the Poincaré residue of $\omega$ along $\left\{x_{i}=0\right\}$ as the restriction to $\left\{x_{i}=0\right\}$ of $a_{i}$. This definition does not depend on the choice of the system of local coordinates (see $[\mathrm{D}]$ ).

Definition 1. Let $X$ be a complex manifold of dimension $2 n-1$ and $Y$ a normal crossings divisor of $X$. We say that a logarithmic differential form $\omega$ of degree 1 is a logarithmic contact form with poles along $Y$ if $\omega(d \omega)^{n-1}$ is a local generator of $\Omega_{X}^{2 n-1}\langle Y\rangle$.

We say that an invertible sub- $\mathcal{O}_{X}$-module $\mathcal{L}$ of $\Omega_{X}^{1}\langle Y\rangle$ is a logarithmic contact structure with poles along $Y$ if $\mathcal{L}$ is locally generated by logaritmic contact forms with poles along $Y$. A logarithmic contact manifold is a pair $(X, \mathcal{L})$ where $X$ is a complex manifold and $\mathcal{L}$ is a logarithmic contact structure on $X$.

Let $\left(X_{1}, \mathcal{L}_{1}\right),\left(X_{2}, \mathcal{L}_{2}\right)$ be two logarithmic contact manifolds. Let $\varphi: X_{1} \rightarrow X_{2}$ be a holomorphic map. We say that $\varphi$ is a contact transformation if for any local generator $\omega$ of $\mathcal{L}_{2}$ the logarithmic differential form $\varphi^{*} \omega$ is a local generator of $\mathcal{L}_{1}$. We say that $\varphi$ is a contact isomorphism if $\varphi$ is an invertible contact transformation.

When $Y$ is the empty set we get the usual definitions of contact manifold and contact transformation. We remark that in the category of logarithmic contact manifolds a contact transformation is not always a local homeomorphism (see Proposition 4.3.1(i)).

It follows from (2) that $\Omega_{X}^{1}\langle Y\rangle$ is a locally free $\mathcal{O}_{X}$-module of $\operatorname{rank} \operatorname{dim} X$. We will denote by $\pi_{Y}: T^{*}\langle X / Y\rangle \rightarrow X$ a vector bundle on $X$ with sheaf of sections isomorphic to $\Omega_{X}^{1}\langle Y\rangle$. This vector bundle is unique up to isomorphism. Let $T^{*} X$ denote the cotangent bundle of $X$. Its sheaf of local sections equals $\Omega_{X}^{1}$. Since $\left.\Omega_{X}^{1}\langle Y\rangle\right|_{X \backslash Y}=\Omega_{X \backslash Y}^{1}$, the vector bundles $T^{*}\langle X / Y\rangle$ and $T^{*} X$ are isomorphic on $X \backslash Y$. We call $T^{*}\langle X / Y\rangle$ the logarithmic cotangent bundle of $X$ with poles along $Y$. 
It is well known that there is a canonical 1-form $\theta$ on $T^{*} X$. We can define in a similar way a canonical logarithmic differential form on $T^{*}\langle X / Y\rangle$, with poles along $\pi_{Y}^{-1}(Y), \theta_{Y}$, such that $\left.\theta_{Y}\right|_{\pi^{-1}(X \backslash Y)}=\left.\theta\right|_{\pi^{-1}(X \backslash Y)}$. Given a system of local coordinates $\left(x_{1}, \ldots, x_{n}\right)$ on an open subset $U$ of $X$, adapted to the divisor $Y$, a canonical system of local coordinates on $\pi_{Y}^{-1}(U)$, adapted to the divisor $Y$, is the system of local coordinates $\left(x_{1}, \ldots, x_{n}, \xi_{1}, \ldots, \xi_{n}\right)$ on $\pi_{Y}^{-1}(U)$ such that

$$
\left.\theta_{Y}\right|_{\pi^{-1}(U)}=\sum_{i=1}^{\nu} \xi_{i} \frac{d x_{i}}{x_{i}}+\sum_{i=\nu+1}^{n} \xi_{i} d x_{i} .
$$

We can easily deduce from (3) that $\left(d \theta_{Y}\right)^{n}$ generates $\left.\Omega_{T^{*}}^{2 n}\langle X / Y\rangle{ }^{\left\langle\pi^{-1}\right.}(Y)\right\rangle$. Because of that we say that $d \theta_{Y}$ is a logarithmic symplectic form with poles along $Y$.

We call a pair $(X, \sigma)$ a logarithmic symplectic manifold if $X$ is a complex manifold and $\sigma$ is a logarithmic symplectic form with poles along some normal crossings divisor $Y$. We call $Y$ the set of poles of $X$.

We will denote by $\pi_{Y}: \mathbf{P}^{*}\langle X / Y\rangle \rightarrow X$ the projective bundle associated to the vector bundle $T^{*}\langle X / Y\rangle$. We call the projective bundle $\mathbf{P}^{*}\langle X / Y\rangle$ the projective logarithmic cotangent bundle of $X$ with poles along $Y$. We will denote by $\gamma$ the canonical map from $T^{*}\langle X / Y\rangle \backslash X$ onto $\mathbf{P}^{*}\langle X / Y\rangle$.

The projective bundle $\mathbf{P}^{*}\langle X / Y\rangle$ is our first example of a logarithmic contact manifold (see Remark 4.1).

Definition 2. Let $(X, \mathcal{L})$ be a logarithmic contact manifold with poles along a normal crossings divisor $Y$. Let $Y_{0}$ be a smooth irreducible component of $Y$. We say that a point $y^{0}$ belongs to the residual submanifold of $X$ along $Y_{0}$ if the residue of $\omega$ along $Y_{0}$ vanishes at $y^{0}$, for any local section $\omega$ of $\mathcal{L}$ defined in a neighbourhood of $y^{0}$. The residual set of $(X, \mathcal{L})$ is the union of the residual submanifolds of $X$. If the divisor $Y$ is smooth, then the residual submanifold of $X$ is the residual submanifold of $X$ along $Y$. We define in a similar way the residual set of a logarithmic symplectic manifold.

TheOREM 1. Let $(X, \mathcal{L})$ be a contact manifold of dimension $2 n-1$ with poles along a normal crossings divisor $Y$. Given $y_{0} \in X$, let $\nu$ be the number of irreducible components of $Y$ at $y_{0}$. Then $\nu \leq n$. Moreover,

(i) If $y_{0}$ belongs to the residual set of $X$, then there is an open subset $U$ of $X$ and a system of local coordinates $\left(x_{1}, \ldots, x_{n}, p_{1}, \ldots, p_{n-1}\right)$ on $U$, centered at $y_{0}$, such that $Y \cap U=\left\{x_{1} \cdots x_{\nu}=0\right\}$ and

is a local generator of $\mathcal{L}$.

$$
d x_{n}-\sum_{i=1}^{\nu} p_{i} \frac{d x_{i}}{x_{i}}-\sum_{i=\nu+1}^{n-1} p_{i} d x_{i}
$$

(ii) If $y_{0}$ does not belong to the residual set of $X$, then there is an open subset $U$ of $X$ and a system of local coordinates $\left(x_{1}, \ldots, x_{n}, p_{1}, \ldots, p_{n-1}\right)$ on $U$, centered at $y_{0}$, such that $Y \cap U=\left\{x_{1} \ldots x_{\nu-1} x_{n}=0\right\}$ and

is a local generator of $\mathcal{L}$.

$$
\frac{d x_{n}}{x_{n}}-\sum_{i=1}^{\nu-1} p_{i} \frac{d x_{i}}{x_{i}}-\sum_{i=\nu}^{n-1} p_{i} d x_{i}
$$


It follows from theorem 1 that the residual submanifold of $X$ along $Y_{0}$ is a submanifold of $Y_{0}$. Moreover, this theorem shows that a contact manifold is locally isomorphic to an open set of a projective logarithmic cotangent bundle. This theorem contains as a particular case the classical Darboux Theorem for contact manifolds.

Definition 3. We say that a submanifold $\Gamma$ of a $\operatorname{logarithmic}$ contact manifold $(X, \mathcal{L})$ is Legendrian if the dimension of $\Gamma$ equals $\frac{1}{2}(\operatorname{dim} X-1)$ and the restriction to $\Gamma$ of a section $\omega$ of $\mathcal{L}$ vanishes for any $\omega$. We say that a subvariety $\Gamma$ of $X$ is Legendrian if its non-singular part is Legendrian.

Let $X$ be a logarithmic contact manifold with poles along a normal crossings divisor $Y$. Let $\Gamma$ be a subvariety of $X$ of dimension $\frac{1}{2}(\operatorname{dim} X-1)$. We say that $\Gamma$ is a Legendrian variety if $\Gamma \cap(X \backslash Y)$ is a Legendrian subvariety of $X \backslash Y$ and $\Gamma$ is the closure in $X$ of $\Gamma \cap(X \backslash Y)$.

Definition 4. Let $M$ be a complex manifold and $C$ a closed submanifold of $X$. We define the conormal bundle $T_{C}^{*} M$ of $X$ along $C$ by the exact sequence

$$
0 \rightarrow T_{C}^{*} M \rightarrow C \times_{M} T^{*} M \rightarrow T^{*} C \rightarrow 0,
$$

where the morphism $C \times{ }_{M} T^{*} M \rightarrow T^{*} C$ is induced by the inclusion map $C \hookrightarrow M$. If $C$ is a subvariety of $M$ we define the conormal bundle $T_{C}^{*} M$ of $M$ along $C$ as the closure in $T^{*} M$ of $T_{C_{\text {reg }}}^{*}\left(M \backslash C_{\text {sing }}\right)$. The projective conormal bundle of $M$ along $C$ is the image $\mathbf{P}_{C}^{*} M$ of $T_{C}^{*} M \backslash M$ by the canonical projection $\gamma: T^{*} M \backslash M \rightarrow \mathbf{P}^{*} M$.

Let $N$ be a normal crossings divisor of $M$ and $C$ a subvariety of $M$ that is the closure in $M$ of a closed subvariety of $M \backslash N$. The logarithmic conormal bundle of $M$ along $C$, with poles along $N$, is the closure in $\mathbf{P}^{*}\langle M / N\rangle$ of the conormal bundle of $N \backslash(N \cap C)$ along $C \backslash(N \cap C)$. We will denote it by $\mathbf{P}_{C}^{*}\langle M / N\rangle$.

Proposition 2. Let $M$ be a complex manifold of dimension 2 and $N$ a normal crossings divisor of $M$. Let $\Gamma$ be an irreducible Legendrian subvariety of $\mathbf{P}^{*}\langle M / N\rangle$. Then $\Gamma$ equals the logarithmic conormal bundle $\mathbf{P}_{\pi(\Gamma)}^{*}\langle M / N\rangle$ of $M$ along $\pi(\Gamma)$, with poles along $N$.

Let $(X, \mathcal{L})$ be a logarithmic contact manifold with poles along a normal crossings divisor $Y$. Let $\Gamma$ be a Legendrian submanifold of $(X, \mathcal{L})$. We say that $Y$ intersects transversally the set of poles of $X$ at $p^{0}$ if $Y$ is smooth at $p^{0}$ and $T_{p^{0}} Y+T_{p^{0}} \Gamma=T_{p^{0}} X$. We say that $\Gamma$ intersects transversally the set of poles of $X$ if $\Gamma$ intersects $Y$ transversally at $p^{0}$ for all $p^{0} \in X \cap \Gamma$.

TheOREM 3. Let $(X, \mathcal{L})$ be a logarithmic contact manifold of dimension 3 with poles along a normal crossings divisor $Y$. Let $\Gamma$ be a Legendrian curve of $(X, \mathcal{L})$. Let $p^{0}$ be a point of $Y \cap \Gamma$.

(i) If $Y$ is smooth at $p^{0}$, then $p^{0}$ belongs to the residual submanifold of $X$.

(ii) If $p^{0}$ belongs to the singular set of $Y$, then $p^{0}$ does not belong to the residual set of $X$.

(iii) If $\Gamma$ is smooth and $\Gamma$ and $\Lambda$ are transversal at a point $p^{0}$, then there is an open subset $U$ of $X$ and a system of local coordinates $(x, y, p)$ on $U$, centered at $p^{0}$, such that $d y-p d x / x$ is a generator of $\left.\mathcal{L}\right|_{U}$ and $\Gamma \cap U=\{y=p=0\}$. 
Let $X$ be a complex manifold. A group action $\alpha: \mathbf{C}^{*} \times X \rightarrow X$ is called a free group action of $\mathbf{C}^{*}$ on $X$ if for each $x \in X$ the isotropy subgroup $\left\{t \in \mathbf{C}^{*}: \alpha(t, x)=x\right\}$ equals $\{1\}$. A manifold $X$ with a free group action $\alpha$ of $\mathbf{C}^{*}$ is called a conic manifold. We associate to each free group action $\alpha$ of $\mathbf{C}^{*}$ on $X$ a vector field $\rho$, the Euler vector field of $\alpha$. Given $\lambda \in \mathbf{C}$, put $\mathcal{O}_{X}(\lambda)=\left\{f \in \mathcal{O}_{X}: \rho f=\lambda f\right\}$. A section $f$ of $\mathcal{O}_{X}(\lambda)$ is called a homogeneous function of degree $\lambda$. Given two conic complex manifolds $\left(X_{1}, \alpha_{1}\right)$ and $\left(X_{2}, \alpha_{2}\right)$, a holomorphic map $\varphi: X_{1} \rightarrow X_{2}$ is called homogeneous if it commutes with the actions $\alpha_{1}, \alpha_{2}$.

Definition 5. Let $X$ be a complex manifold of dimension $2 n$ and $Y$ a normal crossings divisor of $X$. We say that $\sigma \in \Omega_{X}^{2}\langle Y\rangle$ is a logarithmic symplectic form if $\sigma$ is locally exact and $\sigma^{n}$ is a generator of the invertible $\mathcal{O}_{X}$-module $\Omega_{X}^{2 n}\langle Y\rangle$. If $X$ is a conic manifold, then we say that a logarithmic symplectic form $\sigma$ is a homogeneous logarithmic symplectic form if $\alpha_{t}^{*} \sigma=t \sigma$, for any $t \in \mathbf{C}^{*}$. Here $\alpha_{t}$ equals $\alpha(t, *)$.

If $X$ is a [conic] manifold and $\sigma$ is a [homogeneous] logarithmic symplectic form, we call the pair $(X, \sigma)$ a [homogeneous] logarithmic symplectic manifold. If $\left(X_{1}, \sigma_{1}\right),\left(X_{2}, \sigma_{2}\right)$ are [homogeneous] logarithmic symplectic manifolds, then a [homogeneous] holomorphic map $\varphi: X_{1} \rightarrow X_{2}$ is called a morphism of [homogeneous] logarithmic symplectic manifolds if $\varphi^{*} \sigma_{2}=\sigma_{1}$.

We notice that if the normal crossings divisor $Y$ equals the empty set, then we get the usual definition of [homogeneous] symplectic manifold. We remark that a morphism of logarithmic symplectic manifolds is not necessarily a local homeomorphism, as it happens in the category of symplectic manifolds.

If $(X, \sigma)$ is a homogeneous symplectic manifold with poles along $Y$ and $\rho$ is its Euler vector field, then the interior product $\theta=i(\rho) \sigma$ is a logarithmic differential form with poles along $Y$. Moreover, $d \theta$ equals $\sigma$. We call $\theta$ the canonical 1 -form of the homogeneous logarithmic symplectic manifold $(X, \sigma)$.

Definition 6. Given a complex manifold $X$, we say that a $\mathbf{C}$-bilinear morphism $\{\star, \star\}: \mathcal{O}_{X} \times \mathcal{O}_{X} \rightarrow \mathcal{O}_{X}$ is a Poisson bracket if $\{f, g\}=-\{g, f\},\{f g, h\}=f\{g, h\}+$ $g\{f, h\}$ and $\{\{f, g\}, h\}+\{\{g, h\}, f\}+\{\{h, f\}, g\}=0$, for all local sections $f, g, h$ of $\mathcal{O}_{X}$. We call a complex manifold $X$ endowed with a Poisson bracket a Poisson manifold.

An analytical subset $V$ of $X$ is called involutive if $\left\{I_{V}, I_{V}\right\} \subset I_{V}$.

If $\left(X_{1},\{\star, \star\}_{1}\right),\left(X_{2},\{\star, \star\}_{2}\right)$ are Poisson manifolds and $\varphi: X_{1} \rightarrow X_{2}$ is a holomorphic map such that $\left\{\varphi^{*} f, \varphi^{*} g\right\}_{1}=\varphi^{*}\{f, g\}_{2}$, for any holomorphic functions $f, g$ defined in an open set of $X_{2}$, we call $\varphi$ a morphism of Poisson manifolds.

If $V$ is an involutive subvariety of $X_{2}$ and $\varphi: X_{1} \rightarrow X_{2}$ is a morphism of Poisson manifolds then $\varphi^{-1}(V)$ is an involutive subvariety of $X_{1}$.

R e mark 1. A logarithmic symplectic manifold has a canonical structure of Poisson manifold. Given $\alpha \in \Omega_{X}^{1}\langle Y\rangle$, let $H(\alpha)$ denote the only vector field $u \in \Theta_{X}\langle Y\rangle$ such that $\langle u, \alpha\rangle=\sigma(u, H(\alpha))$. The bilinear form $(f, g) \mapsto\langle d g, H(d f)\rangle$ is a Poisson bracket on $\mathcal{O}_{X}$. If $(x, \xi)$ is a system of local coordinates on an open set $U$ of $X$ such that $\left.\sigma\right|_{U}$ equals the 
differential of (3) then $\{f, g\}$ equals

$$
\sum_{i=1}^{\nu} x_{i}\left(\frac{\partial f}{\partial \xi_{i}} \frac{\partial g}{\partial x_{i}}-\frac{\partial f}{\partial x_{i}} \frac{\partial g}{\partial \xi_{i}}\right)+\sum_{i=\nu+1}^{n}\left(\frac{\partial f}{\partial \xi_{i}} \frac{\partial g}{\partial x_{i}}-\frac{\partial f}{\partial x_{i}} \frac{\partial g}{\partial \xi_{i}}\right) .
$$

In particular

$$
\left\{\xi_{i}, x_{j}\right\}= \begin{cases}\delta_{i j} x_{j} & \text { if } 1 \leq j \leq \nu \\ \delta_{i j} & \text { if } \nu+1 \leq j \leq n .\end{cases}
$$

Remark 2. Given a homogeneous logarithmic symplectic manifold $X$, we endow the manifold $\mathbf{X}$ of the orbits of its $\mathbf{C}^{*}$-action with a logarithmic contact structure (see Remark 4.1). Let $\gamma=\gamma_{X}$ denote the canonical map $X \rightarrow \mathbf{X}$. A morphism of homogeneous logarithmic symplectic manifolds $\varphi: X \rightarrow Y$ induces a contact transformation $\varphi: \mathbf{X} \rightarrow \mathbf{Y}$ such that $\gamma_{Y} \varphi=\varphi \gamma_{X}$. The functor $X \mapsto \mathbf{X}$ is an equivalence of categories between the category of homogeneous logarithmic symplectic manifolds and the category of contact manifolds.

Given a logarithmic contact manifold $X$ and a subvariety $V$ of $X$, we say that $V$ is involutive if $\gamma_{X}^{-1}(V)$ is an involutive subvariety of $\widehat{X}$.

3. Systems of meromorphic microdifferential equations. Given a fiber bundle $\tau: E \rightarrow X$, we will denote by $\mathcal{O}_{[E]}$ the subsheaf of $\mathcal{O}_{E}$ of sections wich are polynomial in the fibers of $\tau$.

Let $X$ be a complex manifold and $Y$ a normal crossings divisor of $X$. We will denote by $\mathcal{D}_{X}\langle Y\rangle$ the sub- $\mathcal{O}_{X}$-algebra of $\mathcal{H}_{\mathrm{om}_{\mathbf{X}}}\left(\mathcal{O}_{X}, \mathcal{O}_{X}\right)$ generated by $\Theta_{X}\langle Y\rangle$. We call the elements of $\mathcal{D}_{X}\langle Y\rangle$ differential operators on $X$ tangent to $Y$. Let $j: X \backslash Y \hookrightarrow X$ be the open inclusion. Let $\pi_{Y}: T^{*}\langle X / Y\rangle \rightarrow X$ be the projective cotangent bundle. A section $P$ of $j_{*} j^{-1} \mathcal{D}_{X}$ is called a meromorphic differential operator if there is a holomorphic function $f$ that vanishes at most in $Y$ such that $f P \in \mathcal{D}_{X}$. If $Y$ equals the empty set then the sheaves $\mathcal{D}_{X}\langle Y\rangle$ and $\mathcal{D}_{X}[Y]$ equal the sheaf $\mathcal{D}_{X}$ of germs of differential operators on $X$.

Definition 1. Let $U$ be an open set of $X$ and let $\left(x_{1}, \ldots, x_{n}\right)$ be a system of local coordinates adapted to the normal crossings divisor $Y$. Let $\left(x, \xi^{\prime}\right)$ be the associated system of canonical coordinates on $\pi_{Y}^{-1}(U) \subset T^{*}\langle X / Y\rangle$. Given a section $P$ of $\mathcal{D}_{X}\langle Y\rangle$, we define the total symbol of $P$ as the element $\left(P_{j}\right)$ of $\mathcal{O}_{\left[T^{*}\langle X / Y\rangle\right]}$

$$
e^{-\left\langle x, \xi^{\prime}\right\rangle_{\nu}} P e^{\left\langle x, \xi^{\prime}\right\rangle_{\nu}}
$$

where $\left\langle x, \xi^{\prime}\right\rangle_{\nu}=\sum_{i=1}^{\nu} \xi_{i}^{\prime} \log x_{i}+\sum_{i=\nu+1}^{n} x_{i} \xi_{i}^{\prime}$ and each $P_{j}$ is a homogeneous polynomial of degree $j$ relative to the action of $\mathbf{C}^{*}$ on the fibers of $T^{*}\langle X / Y\rangle$ and coefficients in $\pi_{Y}^{-1} \mathcal{O}_{X}$.

Let $\delta_{x_{i}}$ denote $x_{i} \partial_{x_{i}}$, if $1 \leq i \leq \nu$, and $\partial_{x_{i}}$, if $\nu+1 \leq i \leq n$.

The following proposition tells us how to calculate the total symbol of the sum and product of two differential operators tangent to $Y$. 
Proposition 1. Given two sections $P, Q$ of $\mathcal{D}_{X}\langle Y\rangle,(P+Q)_{l}=P_{l}+Q_{l}$,

$$
(P Q)_{l}=\sum_{\substack{l=j+k-|\alpha| \\ \alpha \in \mathbf{N}^{n}}} \frac{1}{\alpha !}\left(\partial_{\xi}^{\alpha} P_{j}\right)\left(\delta_{x}^{\alpha} Q_{k}\right) .
$$

If $P \neq 0$ then the principal symbol of $P$ relative to $Y$ is the homogeneous part $\sigma_{Y}(P)$ of highest degree of the total symbol relative to $Y$ and to some system of local coordinates.

Let $X_{n}$ be a copy of $\mathbf{C}^{n}$ with coordinates $\left(x_{1}, \ldots, x_{n}\right)$. Put $Y=\left\{x_{1}=0\right\}$. Let $\theta=\sum_{i=1}^{n} \xi_{i} d x_{i}$ be the canonical 1-form of $T^{*} X$. Let $\theta^{\prime}=\xi_{1}^{\prime} d x_{1} / x_{1}+\sum_{i=2}^{n} \xi_{i}^{\prime} d x_{i}$ be the canonical 1-form of $T^{*}\langle X / Y\rangle$. The following equalities hold:

$$
\sigma\left(x_{1} \partial_{x_{1}}\right)=x_{1} \xi_{1}, \quad \sigma_{Y}\left(x_{1} \partial_{x_{1}}\right)=\xi_{1}^{\prime}, \quad \sigma\left(\partial_{x_{1}}\right)=\xi_{1} .
$$

DEFINITION 2. Let $n$ be a positive integer. Let $\nu$ be a nonnegative integer smaller or equal then $n$. Let $X_{n}$ be a copy of $\mathbf{C}^{n}$ with coordinates $\left(x_{1}, \ldots, x_{n}\right)$. Let $Y_{\nu}$ be the normal crossings divisor $\left\{x_{1} \cdots x_{\nu}=0\right\}$ of $X_{n}$. Let $U$ be an open set of $T^{*}\left\langle X_{n} / Y_{\nu}\right\rangle$. Let $m$ be an integer. Let $\mathcal{E}_{\left(x_{1}, \ldots, x_{n}, \nu\right)}(m)(U)$ be the complex vector space of formal series $\sum_{j \leq m} P_{j}$, where $P_{j}$ is a section of $\mathcal{O}_{T^{*}}\left\langle X_{n} / Y_{\nu}\right\rangle(j)$ on $U$, such that for any compact set $K$ contained in $U$ there is a real number $C$ such that $\sup _{K}\left|P_{-j}\right| \leq C^{j} j$ !, for each $j \geq 0$.

Given $P, Q \in \mathcal{E}_{\left(x_{1}, \ldots, x_{n}, \nu\right)}(m)(U)$, we define their product by $(2)$. The ring $\mathcal{E}_{\left(x_{1}, \ldots, x_{n}, \nu\right)}$ is endowed in this way with a structure of filtered $\mathbf{C}$-algebra.

Given a complex manifold $X$ and a normal crossings divisor $Y$ of $X$, we can associate in this way a canonical sheaf $\mathcal{E}_{\langle X / Y\rangle}$ on $T^{*}\langle X / Y\rangle$ that equals $\mathcal{E}_{X}$ on $T^{*}(X \backslash Y)$. We call this sheaf the sheaf of logarithmic microdifferential operators on $X$ with poles along $Y$ (see $[\mathrm{N} 1])$.

Given a section $P$ of $\mathcal{E}_{\langle X / Y\rangle}$, we can consider a total symbol $\left(P_{j}\right) \in \mathcal{E}_{\left(x_{1}, \ldots, x_{n}, \nu\right)}$ representing $P$ as the "power expansion" of $P$ relative to the system of local coordinates $\left(x_{1}, \ldots, x_{n}\right)$. From now on we will identify the sheaves $\mathcal{E}_{\left(x_{1}, \ldots, x_{n}, \nu\right)}$ and $\mathcal{E}_{\left\langle X_{n} / Y_{\nu}\right\rangle}$.

The ring $\mathcal{E}_{X}$ has no nontrivial two-sided ideals. This is not the case with $\mathcal{E}_{\langle X / Y\rangle}$. The ring $\mathcal{E}_{\langle X / Y\rangle}$ is a $\pi_{Y}^{-1} \mathcal{D}_{X}\langle Y\rangle$-module. In particular, we have a canonical imersion $\pi_{Y}^{-1} I_{Y} \hookrightarrow \mathcal{E}_{\langle X / Y\rangle}$. The two-sided ideal generated by $\pi^{-1} I_{Y}$ is proper. We will denote it by $\mathcal{I}_{Y}$. We notice that, if $f$ is a local generator of $I_{Y}$,

$$
f \mathcal{E}_{\langle X / Y\rangle}=\mathcal{E}_{\langle X / Y\rangle} f=\mathcal{I}_{Y}
$$

Definition 3. Let $j: T^{*}(X \backslash Y) \hookrightarrow T^{*}\langle X / Y\rangle$ be the open inclusion. Given $P \in$ $j_{*} j^{-1} \mathcal{E}_{\langle X / Y\rangle}$, we will say that $P$ is a meromorphic microdifferential operator if, given a local generator $f$ of $\mathcal{I}_{Y}$,

$$
f^{n} P \in \mathcal{E}_{\langle X / Y\rangle}, \quad \text { for } n \gg 0 .
$$

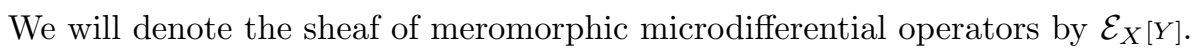

The sheaf $\mathcal{E}_{X}[Y]$ is a sheaf of rings. Given $P, Q \in \mathcal{E}_{X}[Y]$, there are $m, n \geq 0$ such that $f^{m} P, f^{n} Q \in \mathcal{E}_{\langle X / Y\rangle}$. Since $f^{n}\left(f^{m} P\right)=P^{\prime} f^{n}$, for some $P^{\prime} \in \mathcal{E}_{\langle X / Y\rangle}, f^{m+n}(P Q)=$ $P^{\prime}\left(f^{n} Q\right) \in \mathcal{E}_{\langle X / Y\rangle}$.

ThEOREM 4. (i) The sheaves $\mathcal{E}_{\langle X / Y\rangle}$ and $\mathcal{E}_{X[Y]}$ are (left and right) noetherian Rings with zariskian fibers. 
(ii) The following equalities hold:

$$
\left.\mathcal{E}_{\langle X / Y\rangle}\right|_{\pi_{Y}^{-1}(X \backslash Y)}=\left.\mathcal{E}_{X}[Y]\right|_{\pi_{Y}^{-1}(X \backslash Y)}=\left.\mathcal{E}_{X}\right|_{\pi_{Y}^{-1}(X \backslash Y)}
$$

(iii) There are canonical flat imersions

$$
\pi_{Y}^{-1} \mathcal{D}_{X}\langle Y\rangle \hookrightarrow \mathcal{E}_{\langle X / Y\rangle}, \quad \pi_{Y}^{-1} \mathcal{D}_{X}[Y] \hookrightarrow \mathcal{E}_{X}[Y]
$$

Moreover, $\left.\mathcal{E}_{\langle X / Y\rangle}\right|_{X}=\mathcal{D}_{X}\langle Y\rangle,\left.\mathcal{E}_{X}[Y]\right|_{X}=\mathcal{D}_{X}[Y]$.

(iv) If the principal symbol of $P \in \mathcal{E}_{\langle X / Y\rangle, p}$ does not vanish at $p$, then $P$ is invertible.

Pr o of. The results on $\mathcal{E}_{\langle X / Y\rangle}$ were proved in [N1]. The results on $\mathcal{E}_{X}[Y]$ are straightforward consequences.

Since the sheaf $\mathcal{E}_{X}[Y]$ is locally constant along the fibers of $\gamma$, we can identify it with its direct image into $\mathbf{P}^{*}\langle X / Y\rangle$.

Definition 4. Let $X$ be a logarithmic contact manifold. A logarithmic quantization of $X$ [meromorphic quantization of $X]$ is a sheaf of filtered $\mathbf{C}$-algebras $\mathcal{E}$ on $X$ such that for all $p^{0} \in X$ there are a complex manifold $M$, a normal crossings divisor $N$ of $M$, an injective contact transformation $\varphi$ from an open neighbourhood $U$ of $p^{0}$ onto an open set $V$ of $\mathbf{P}^{*}\langle M / N\rangle$ and an isomorphism of filtered $\mathbf{C}$-algebras

$$
\Phi:\left.\varphi_{*}\left(\left.\mathcal{E}\right|_{U}\right) \rightarrow \mathcal{E}_{\langle M / N\rangle}\right|_{V} \quad\left[\Phi:\left.\varphi_{*}\left(\left.\mathcal{E}\right|_{U}\right) \rightarrow \mathcal{E}_{M[N]}\right|_{V}\right]
$$

such that $\sigma(\Phi(P))=\sigma(P) \circ \varphi$, for all sections $P$ of $\varphi_{*}\left(\mathcal{E}_{U}\right)$.

A quantized logarithmic contact manifold [quantized meromorphic contact manifold] is a pair $(X, \mathcal{E})$, where $X$ is a contact manifold and $\mathcal{E}$ is a logarithmic quantization [meromorphic quantization] of $X$.

Let $\left(X_{1}, \mathcal{E}_{1}\right),\left(X_{2}, \mathcal{E}_{2}\right)$ be two quantized logarithmic contact manifolds [quantized meromorphic contact manifolds]. Let $\varphi: X_{2} \rightarrow X_{1}$ be an injective contact transformation. We say that an isomorphism of filtered C-algebras $\Phi: \varphi_{*} \mathcal{E}_{2} \rightarrow \mathcal{E}_{1}$ is a quantization of $\varphi$ if $\sigma(\Phi(P))=\sigma(P) \circ \varphi$, for all sections $P$ of $\varphi_{*} \mathcal{E}_{2}$.

A quantized contact transformation from $\left(X_{1}, \mathcal{E}_{1}\right)$ into $\left(X_{2}, \mathcal{E}_{2}\right)$ is a pair $(\varphi, \Phi)$, where $\varphi$ is a contact transformation and $\Phi: \varphi_{*} \mathcal{E}_{2} \rightarrow \mathcal{E}_{1}$ is a quantization of $\varphi$.

Proposition 5. Let $(X, \mathcal{E})$ be a quantized meromorphic contact manifold with poles along a normal crossings divisor $Y$. Let $\mathcal{M}$ be a coherent $\mathcal{E}$-module. The support of $\mathcal{M}$ is the closure in $X$ of its intersection with $X \backslash Y$. Hence the support of $\mathcal{M}$ is an involutive subvariety of $X$.

Proof. Let $u$ be a section of $\mathcal{M}$ with support contained in $Y$. Then locally there is a local generator $f$ of $I_{Y}$ such that $f^{n} u=0$, for $n$ large enough. Hence $u=0$.

Definition 5. Let $\mathcal{M}$ be a coherent $\mathcal{D}_{X}[Y]$-module. We say that $\mathcal{M}$ is a holonomic

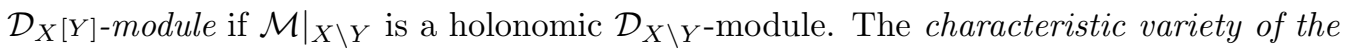
$\mathcal{D}_{X}[Y]$-module $\mathcal{M}$ is the closure in $T^{*}\langle X / Y\rangle$ of $\operatorname{Char}\left(\left.\mathcal{M}\right|_{X \backslash Y}\right)$. We will denote the characteristic variety of the $\mathcal{D}_{X}[Y]$-module $\mathcal{M}$ by $\operatorname{Char}_{Y}(\mathcal{M})$.

Let $(X, \mathcal{E})$ be a quantized meromorphic contact manifold. A system of meromorphic microdifferential equations of $(X, \mathcal{E})$ is a coherent $\mathcal{E}$-module. A holonomic system of mero- 
morphic microdifferential equations is a coherent $\mathcal{E}$-module whose support is a Legendrian variety.

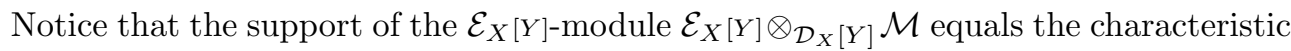
variety of the $\mathcal{D}_{X}[Y]$-module $\mathcal{M}$.

4. Blow up of a [meromorphic] system of microdifferential equations. We will study in this section three types of blow ups that will be needed in the next section in order to win the desingularization game.

Let $U, V$ be two open sets of a complex manifold $X$. Two differential forms $\alpha, \beta$ defined respectively in $U$ and $V$ are said to be equivalent if there is a nowhere vanishing holomorphic function $\varphi$ defined on $U \cap V$ such that $\left.\alpha\right|_{U \cap V}=\left.\varphi \beta\right|_{U \cap V}$. To give a logarithmic contact structure on $X$ with poles along $Y$ is equivalent to giving an open covering $\left(U_{i}\right)$ of $X$ and for each $i$ a logarithmic contact form $\omega_{i}$ on $U_{i}$, with poles along $U_{i} \cap Y$, such that $\omega_{i}$ and $\omega_{j}$ are equivalent, for all pairs $i, j$.

Definition 1. An affine logarithmic contact manifold of dimension $2 n+1$ is a triple

$$
\left(E,\left(a_{1}, \ldots, a_{n+1}, b_{1}, \ldots, b_{n+1}\right), \omega\right)
$$

where $E$ is a complex vector space of dimension $2 n+1, a_{1}, \ldots, a_{n}, b_{1}, \ldots, b_{n+1}$ are holomorphic functions on $E, \omega$ is a meromorphic differential form on $E$ and there is an integer $\nu$ such that

$$
\omega=\sum_{i=1}^{\nu} b_{i} \frac{d a_{i}}{a_{i}}+\sum_{i=\nu+1}^{n} b_{i} d a_{i} .
$$

We will denote (1) by [ $\omega]$. If $\nu=0$, then we call (1) an affine contact manifold.

R e mark 1. Let us denote by $\gamma$ the canonical map from $T^{*}\langle X / Y\rangle \backslash X$ onto $\mathbf{P}^{*}\langle X / Y\rangle$. Suppose for instance that $X$ equals $\mathbf{C}^{2}$ and $Y$ equals $\left\{x_{1}=0\right\}$. In this case, $\theta$ equals $\xi_{1} d x_{1} / x_{1}+\xi_{2} d x_{2}$. The open sets $U_{i}=\gamma\left(\left\{\xi_{i} \neq 0\right\}\right), i=1,2$, cover $\mathbf{P}^{*}\langle X / Y\rangle$. Put $p=$ $-\xi_{1} / \xi_{2}, q=-\xi_{2} / \xi_{1}$. The differential forms $\frac{1}{\xi_{i}} \theta, i=1,2$, define on $\mathbf{P}^{*}\langle X / Y\rangle$ differential forms $\omega_{i}, i=1,2$, where

$$
\omega_{1}=d x_{2}-p \frac{d x_{1}}{x_{1}} \quad \text { and } \quad \omega_{2}=\frac{d x_{1}}{x_{1}}-q d x_{2} .
$$

Since $\left.\omega_{1}\right|_{U_{1} \cap U_{2}}=\left.p_{1} \omega_{2}\right|_{U_{1} \cap U_{2}}$, these logarithmic contact forms define on $\mathbf{P}^{*}\langle X / Y\rangle$ a structure of logarithmic contact manifold. We have thus shown that $\mathbf{P}^{*}\langle X / Y\rangle$ is the obvious patching of the affine logarithmic contact manifolds

$$
\left[d x_{2}-p \frac{d x_{1}}{x_{1}}\right] \text { and }\left[d x_{1}-q \frac{d x_{2}}{x_{2}}\right]
$$

We can show in a similar way that if $Y$ equals $\{x y=0\}$, then $\mathbf{P}^{*}\langle M / N\rangle$ equals the obvious patching of the affine logarithmic contact manifolds

$$
\left[\frac{d x_{2}}{x_{2}}-p \frac{d x_{1}}{x_{1}}\right] \text { and }\left[\frac{d x_{1}}{x_{1}}-q \frac{d x_{2}}{x_{2}}\right]
$$

patched by $p=\frac{1}{q}$. Moreover, $\mathbf{P}^{*} M$ is the patching of the affine contact manifolds

$$
\left[d x_{2}-p d x_{1}\right] \text { and }\left[d x_{1}-q d x_{2}\right] .
$$


Let $M$ be a complex manifold of dimension $n$ and $N$ a normal crossings divisor of $M$. Let $x^{0}$ be a point of $N$. Let $\sigma: \widetilde{M} \rightarrow M$ be the blow up of $M$ along $x^{0}$. Let $E$ be the exceptional divisor of $\sigma$. Put $N^{\sigma}=\sigma^{-1}(N)$. The morphism $\sigma^{-1} \mathcal{D}_{M}[N] \rightarrow \mathcal{D}_{\widetilde{M}^{\left[N^{\sigma}\right]}}$ is flat.

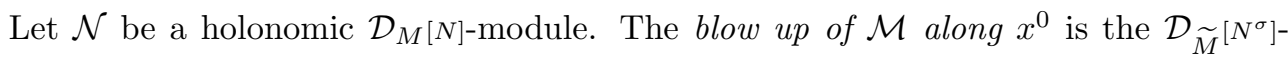

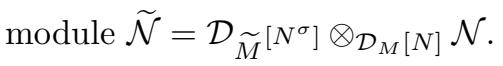

The blow up of a holonomic system is holonomic.

4.1. Let $(X, \mathcal{L})$ be a contact manifold of dimension 3. Let $\Lambda$ be a closed Lagrangian submanifold of $X$. Let $\tau: \widetilde{X} \rightarrow X$ be the blow up of $X$ along $\Lambda$. Let $\widetilde{E}$ be the exceptional divisor of $\tau$. Let $j: \widetilde{X} \backslash E \hookrightarrow \widetilde{X}$ be the inclusion map. Let $\mathcal{O}_{(E)}$ be the subsheaf of $j_{*} j^{-1} \mathcal{O}_{X}$ of sections $f$ such that $f g$ is holomorphic for every function $g$ in $I_{E}$.

Proposition 1.1. (i) The $\mathcal{O}_{\tilde{X}}$-module $\widetilde{\mathcal{L}}=\mathcal{O}_{(E)} \tau^{*} \mathcal{L}$ is a structure of logarithmic contact manifold with poles along $E$. Moreover, $\left.\tau\right|_{\widetilde{X} \backslash E}: \widetilde{X} \backslash E \rightarrow X$ is a contact transformation.

(ii) The proper inverse image of a Legendrian curve of $X$ is a Legendrian curve of $\tilde{X}$.

Definition 1.1. We call the pair $(\tau: \tilde{X} \rightarrow X, \widetilde{\mathcal{L}})$ the blow up of the contact manifold $(X, \mathcal{L})$ along its Legendrian submanifold $\Lambda$.

TheOREM 1.2. Let $(X, \mathcal{E})$ be a quantized contact manifold of dimension 3 . Let $\Lambda$ be a Lagrangian submanifold of $X$. Let $\tau: \widetilde{X} \rightarrow X$ be the blow up of $X$ along $\Lambda$. Let $E$ be the exceptional divisor of $\tau$.

(i) There is a meromorphic quantization $\widetilde{\mathcal{E}}$ of the logarithmic contact manifold $\tilde{X}$ and a morphism of filtered $\mathbf{C}$-algebras

$$
\Phi: \tau^{-1} \mathcal{E} \rightarrow \widetilde{\mathcal{E}}
$$

such that $\left.\Phi\right|_{\widetilde{X} \backslash E}:\left.\left.\tau^{-1} \mathcal{E}\right|_{\widetilde{X} \backslash E} \rightarrow \widetilde{\mathcal{E}}\right|_{\widetilde{X} \backslash E}$ is an isomorphism. The morphism $\Phi$ is flat.

Given another quantization $\mathcal{E}^{\prime}$ of $\widetilde{X}_{0}$ and another morphism of filtered $\mathbf{C}$-algebras $\Phi^{\prime}: \tau^{-1} \mathcal{E} \rightarrow \mathcal{E}^{\prime}$ such that $\left.\Phi^{\prime}\right|_{\widetilde{X} \backslash E}:\left.\left.\tau^{-1} \mathcal{E}\right|_{\widetilde{X} \backslash E} \rightarrow \mathcal{E}^{\prime}\right|_{\widetilde{X} \backslash E}$ is an isomorphism, there is one and only one isomorphism of filtered $\mathbf{C}$-algebras $\Psi: \widetilde{\mathcal{E}} \rightarrow \mathcal{E}^{\prime}$ such that $\Psi \Phi=\Phi^{\prime}$.

(ii) Given a holonomic $\mathcal{E}$-module $\mathcal{M}$ the $\widetilde{\mathcal{E}}$-module $\widetilde{\mathcal{M}}=\widetilde{\mathcal{E}} \otimes_{\mathcal{E}} \mathcal{M}$ is also holonomic. Moreover, the support of $\widetilde{\mathcal{M}}$ equals the proper inverse image of the support of $\mathcal{M}$.

We refer to $(1.1)$ as the blow up of the quantized contact manifold $(X, \mathcal{E})$ along $\Lambda$. We call $\widetilde{\mathcal{M}}$ the blow up of the holonomic $\mathcal{E}$-module $\mathcal{M}$ along $\Lambda$.

Let $M$ be a complex manifold. Let $\mathcal{N}$ be a coherent $\mathcal{D}_{M}$-module. An hypersurface $H$ of $M$ is called noncharacteristic to $\mathcal{N}$ if $T_{H}^{*} M \cap \operatorname{Char} \mathcal{N} \subset T_{M}^{*} M$.

TheOREM 1.3. Let $M$ be a complex manifold of dimension 2. Let $Z$ be a discrete subset of $M$. Let $\sigma: \widetilde{M} \rightarrow M$ be the blow up of $\widetilde{M}$ along $Z$. Let $E$ be the exceptional divisor of $\sigma$. Put $\Lambda=\pi_{M}^{-1}(Z) \subset \mathbf{P}^{*} M$.

(i) The blow up of the contact manifold $\mathbf{P}^{*} M$ along $\Lambda$ equals the logarithmic contact 
manifold $\mathbf{P}^{*}\langle\widetilde{M} / E\rangle$. Moreover, the diagram below commutes.

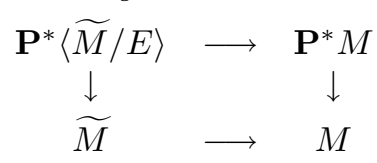

(ii) Let $H$ be an hypersurface of $M$. Let $Z$ be a discrete subset of $H$. Let $H^{\sigma},[\widetilde{H}]$ be the inverse image [proper inverse image] of $H$ by $\sigma$. Put $M^{\prime}=\widetilde{M} \backslash \widetilde{H}, E^{\prime}=E \backslash \widetilde{H}$. Given a holonomic $\mathcal{D}_{M}$-module $\mathcal{N}$ such that $H$ is noncharacteristic to $\mathcal{N}$ and the intersection of $H$ with the singular support of $\mathcal{N}$ equals $Z$, the support of the blow up of $\mathcal{E}_{M} \otimes_{\mathcal{D}_{M}} \mathcal{N}$ along $\Lambda$ is contained in $\mathbf{P}^{*}\left\langle M^{\prime} / E^{\prime}\right\rangle$. Moreover,

$$
\left.\left.\mathcal{E}_{\tilde{M}}\left[H^{\sigma}\right] \otimes_{\mathcal{D}_{\tilde{M}}\left[H^{\sigma}\right]}\left(\mathcal{D}_{M}[H] \otimes_{\mathcal{D}_{M}} \mathcal{N}\right)^{\sim}\right|_{\mathbf{P}^{*}\left\langle M^{\prime} / E^{\prime}\right\rangle} \stackrel{\sim}{\longrightarrow}\left(\mathcal{E}_{M} \otimes_{\mathcal{D}_{M}} \mathcal{N}\right)^{\sim}\right|_{\mathbf{P}^{*}\left\langle M^{\prime} / E^{\prime}\right\rangle} .
$$

Statement 1.3(ii) says that the blow up of the microlocalization of a $\mathcal{D}_{M}$-module $\mathcal{N}$ along the inverse image of a discrete subset $Z$ of $M$ is essentially a microlocalization of the blow up along $Z$ of the localization of $\mathcal{N}$ along a noncharacteristic curve.

We recall a classical theorem of contact geometry (see for instance $[\mathrm{H}]$ ).

THEOREM 1.4. Let $(X, \mathcal{L})$ be a contact manifold and let $\Gamma$ be a Legendrian submanifold of $X$. If $y_{0} \in \Gamma$, then there is an open neighbourhood $U$ of $y_{0}$ and a system of local coordinates $\left(x_{1}, \ldots, x_{n}, p_{1}, \ldots, p_{n-1}\right)$, centered at $y_{0}$, such that

$$
\Gamma \cap U=\left\{x_{1}=\cdots=x_{n}=0\right\}
$$

and $d x_{n}-\sum_{i=1}^{n-1} p_{i} d x_{i}$ generates $\mathcal{L}$ on $U$.

Statements 1.1(i) and 1.3(i) were proved in [N1]. Statement 1.1(ii) follows immediately from the definitions of proper inverse image and of Legendrian subvariety of a logarithmic contact manifold. Statement 1.2(ii) follows immediately from the definitions of blow up of a holonomic system along a Legendrian submanifold and Proposition 3.5.

We will now sketch the proofs of 1.1(i) and 1.3(i). Let $M$ be a complex manifold and let $Z$ be a discrete subset of $M$. Put $X=\mathbf{P}^{*} M$ and $\Lambda=\pi^{-1}(Z)$. Let us show that there is a canonical map $\tau: \mathbf{P}^{*}\langle\tilde{M} / E\rangle \rightarrow \mathbf{P}^{*} M$ such that the diagram below commutes.

$$
\begin{array}{ccccc}
\mathbf{P}^{*}(\widetilde{M} \backslash E) & \hookrightarrow & \mathbf{P}^{*}\langle\widetilde{M} / E\rangle & \rightarrow & \widetilde{M} \\
\| & & \downarrow & & \downarrow \\
\mathbf{P}^{*}(M \backslash Z) & \hookrightarrow & \mathbf{P}^{*} M & \rightarrow & M
\end{array}
$$

Since the problem is local in $M$ we may assume that $M$ is a copy of $\mathbf{C}^{2}$ with coordinates $(x, y)$. Put $x_{0}=x, y_{0}=y / x, x_{1}=x / y, y_{1}=y$. The manifold $\widetilde{M}$ is the obvious patching of the copies $M_{0}, M_{1}$ of $\mathbf{C}^{2}$ with coordinates $(x, y / x),(x / y, y)$. Moreover, $E_{0}:=E \cap M_{0}=$ $\left\{x_{0}=0\right\}, E_{1}:=E \cap M_{1}=\left\{y_{1}=0\right\}$. The difeomorphism $\tilde{M} \backslash E \rightarrow M \backslash Z$ induces a symplectic transformation

$$
T^{*}(\tilde{M} \backslash E) \rightarrow T^{*}(M \backslash Z) .
$$

Let $\left(x_{0}, y_{0} ; \xi_{0}, \eta_{0}\right)$ be the canonical coordinates associated to $\left(x_{0}, y_{0}\right)$. The symplectic transformation (1.4) takes $\left(x_{0}, y_{0} ; \xi_{0}, \eta_{0}\right)$ into

$$
\left(x_{0}, x_{0} y_{0} ; \xi_{0}-\frac{y_{0}}{x_{0}} \eta_{0}, \frac{1}{x_{0}} \eta_{0}\right) .
$$


Let $\left(x_{0}, y_{0} ; \xi_{0}^{\prime}, \eta_{0}^{\prime}\right)$ be the logarithmic canonical coordinates associated to $\left(x_{0}, y_{0}\right)$ relative to $\left\{x_{0}=0\right\}$. Notice that $\xi_{0}^{\prime}=x_{0} \xi_{0}, \eta_{0}^{\prime}=\eta_{0}$. The symplectic transformation considered above is now given by

$$
\left(x_{0}, y_{0} ; \xi_{0}^{\prime}, \eta_{0}^{\prime}\right) \mapsto\left(x_{0}, x_{0} y_{0} ; \frac{1}{x_{0}}\left(\xi_{0}^{\prime}-y_{0} \eta_{0}^{\prime}\right), \frac{1}{x_{0}} \eta_{0}^{\prime}\right) .
$$

Therefore we have a meromorphic map from $T^{*}\left\langle M_{0} / E_{0}\right\rangle$ into $T^{*} M\left[\mathbf{P}^{*}\left\langle M_{0} / E_{0}\right\rangle\right.$ into $\left.\mathbf{P}^{*} M\right]$. If we consider $\left(\xi_{0}, \eta_{0}\right)$ and $\left(\xi_{0}^{\prime}, \eta_{0}^{\prime}\right)$ as homogeneous coordinates, the morphism above is still given by

$$
\left(x_{0}, y_{0} ; \xi_{0}^{\prime}, \eta_{0}^{\prime}\right) \mapsto\left(x_{0}, x_{0} y_{0} ; \xi_{0}^{\prime}-y_{0} \eta_{0}^{\prime}, \eta_{0}^{\prime}\right)
$$

Since $\left(\xi_{0}^{\prime}-y_{0} \eta_{0}^{\prime}, \eta_{0}^{\prime}\right)=(0,0)$ if and only if $\left(\xi_{0}^{\prime}, \eta_{0}^{\prime}\right)=(0,0)$, the meromorphic map (1.5) is actually holomorphic. We leave to the reader the verification that the map $\tau$ is the blow up of $\mathbf{P}^{*} M$ along $\Lambda$, proving 1.3(i). Statement 1.1(i) follows from Theorem 1.4 and 1.3(i).

We will now prove 1.2(i). There is a canonical isomorphism of filtered $\mathbf{C}$-algebras

$$
\Phi_{0}:\left.\left.\tau^{-1} \mathcal{E}_{M}\right|_{\mathbf{P}^{*}(\widetilde{M} \backslash E)} \rightarrow \mathcal{E}_{\widetilde{M}^{[E]}}\right|_{\mathbf{P}^{*}(\widetilde{M} \backslash E)} .
$$

The pair $\left(\left(\left.\tau\right|_{\mathbf{P}^{*}(\widetilde{M} \backslash E)}\right)^{-1}, \Phi_{0}\right)$ is a quantized contact transformation. Let $j: \mathbf{P}^{*}(\widetilde{M} \backslash E) \rightarrow$ $\mathbf{P}^{*}\langle\widetilde{M} / E\rangle$ be the inclusion map. The morphism $\Phi_{0}$ induces a morphism

$$
\Phi: \tau^{-1} \mathcal{E}_{M} \rightarrow j_{*} j^{-1} \mathcal{E}_{\widetilde{M}^{[E]}}
$$

It suffices to show that the image of $\Phi$ is contained in $\mathcal{E}_{\widetilde{M}^{[E]}}$. Given a section $P \in$ $\Gamma\left(U, \tau^{-1} \mathcal{E}_{M}\right)$, we have to show that there is $Q \in \Gamma\left(U, \mathcal{E}_{\widetilde{M}^{[E]}}\right)$ such that $\Phi_{0}\left(\left.P\right|_{U \backslash E}\right)=$ $\left.Q\right|_{U \backslash E}$. Since the problem is local we may assume that $M$ is a copy of $\mathbf{C}^{2}$ with coordinates $(x, y)$ and $U$ is contained in $\mathbf{P}^{*}\left\langle M_{0} / E_{0}\right\rangle$. The restriction of $\Phi_{0}$ to $\mathbf{P}^{*}\left(M_{0} \backslash E_{0}\right)$ is the only quantized contact transformation such that

$$
\Phi_{0}(x)=x_{0}, \quad \Phi_{0}(y)=x_{0} y_{0}, \quad \Phi_{0}\left(\partial_{y}\right)=\frac{1}{x_{0}} \partial_{y_{0}}, \quad \Phi_{0}\left(\partial_{x}\right)=\frac{1}{x_{0}}\left(\delta_{x_{0}}-y_{0} \partial_{y_{0}}\right) .
$$

Let $C$ be a copy of $\mathbf{C}^{4}$ with coordinates $\left(x, y, x_{0}, y_{0}\right)$. Let us consider in $T^{*}\left\langle C /\left\{x_{0}=\right.\right.$ $0\}\rangle$ the associated system of logarithmic symplectic coordinates $\left(x, y, x_{0}, y_{0} ; \xi, \eta_{i}, \xi_{0}, \eta_{0}\right)$. Let $W$ be the open set of $T^{*}\left\langle C /\left\{x_{0}=0\right\}\right\rangle$ where $\eta$ and $\eta_{0}$ do not vanish. Let $p, q$ be the restrictions to $W$ of the canonical projections of $W$ into $T^{*} M$ and $T^{*}\left\langle M_{0} /\left\{x_{0}=0\right\}\right.$. Let $\mathcal{I}$ be the ideal of $\left.\mathcal{E}_{\mathbf{C}^{4}\left[x_{0}=0\right]}\right|_{W}$ generated by

$$
x-x_{0}, \quad y-x_{0} y_{0}, \quad \partial_{y}-\frac{1}{x_{0}} \partial_{y_{0}}, \quad \partial_{x}-\frac{1}{x_{0}}\left(\delta_{x_{0}}-y_{0} \partial_{y_{0}}\right) .
$$

It is enough to show that, given a section $P$ of $p^{-1} \mathcal{E}_{M}$, there is a section $Q$ of $q^{-1} \mathcal{E}_{M_{0}\left[E_{0}\right]}$ such that $P-Q \in \mathcal{I}$. Actually,

$$
\Phi_{0}\left(\left.P\right|_{p(W) \cap \mathbf{P}^{*}(M \backslash Z)}\right)=\left.Q\right|_{q(W) \cap \mathbf{P}^{*}\left(M_{0} \backslash E_{0}\right)}
$$

(see for instance $[\mathrm{S}]$, section I.5). Let us assume that $P$ has order $\leq 0$. Dividing $P$ successively by $x-x_{0}, y-x_{0} y_{0}, \partial_{x} \partial_{y}^{-1}-\left(\delta_{x_{0}} \partial_{y_{0}}^{-1}-y_{0}+\partial_{y_{0}}^{-1}\right)$ (see the division theorem for logarithmic microdifferential operators of [N1]) we may assume that there is a logarithmic microdifferential operator $Q^{\prime}$ with total symbol $\sum_{k \geq 1} \alpha_{k} \eta^{-k}$ such that $P-Q^{\prime} \in \mathcal{I}$ and 
the $\alpha_{k}$ are sections of $q^{-1} \mathcal{O}_{T^{*}\left\langle M_{0} / E_{0}\right\rangle}(0)$. Now $\sum_{k \geq 1} \alpha_{k} x_{0}^{k} \eta_{0}^{-k}$ is a section of $q^{-1} \mathcal{E}_{M_{0}}\left[E_{0}\right]$ and

If $P$ has order $m$ then

$$
\sum_{k \geq 1} \alpha_{k} \eta^{-k}-\sum_{k \geq 1} \alpha_{k} x_{0}^{k} \eta_{0}^{-k} \in \mathcal{I}
$$

$$
\Phi_{0}(P)=\Phi_{0}\left(\partial_{y}^{m}\left(\partial_{y}^{-m} P\right)\right)=\left(\frac{1}{x_{0}}\right)^{m} \partial_{y_{0}}^{m} \Phi\left(\partial_{y}^{-m} P\right) \in q^{-1} \mathcal{E}_{M_{0}}\left[E_{0}\right] .
$$

Statement 1.3(ii) is not used in the proof of the main theorem. Hence its proof its omitted (see [N3]).

4.2. Let $M$ be a complex manifold, let $N$ be a normal crossings divisor of $M$ and let $S$ be a smooth hypersurface of $M$. Let $x^{0}$ be a point of $M$. We say that $S$ intersects $N$ transversally at $x^{0}$ if $x^{0}$ is a regular point of $N$ and $S$ is transversal to $N$ at $x^{0}$. We say that $S$ intersects $N$ transversally if $S$ intersects $N$ transversally at all points $x^{0}$ of $M$.

Let $(X, \mathcal{L})$ be a logarithmic contact manifold of dimension 3 , with poles along a normal crossings divisor $Y$. Let $\Lambda$ be a closed Legendrian submanifold of $X$, tranversal to $Y$. Let $\tau: \widetilde{X} \rightarrow X$ be the blow up of $X$ along $\Lambda$. Let $E$ be the exceptional divisor of $\tau$. Let $j: \widetilde{X} \backslash E \hookrightarrow \widetilde{X}$ be the inclusion map. Put $Y^{\tau}=\tau^{-1}(Y)$.

Proposition 2.1. (i) The $\mathcal{O}_{\widetilde{X}}$-module $\widetilde{\mathcal{L}}=\mathcal{O}_{(E)} \tau^{*} \mathcal{L}$ is a structure of logarithmic contact manifold with poles along $Y^{\tau}$. Moreover, $\left.\tau\right|_{\widetilde{X} \backslash E}: \widetilde{X} \backslash E \rightarrow X$ is a contact transformation.

(ii) The proper inverse image of a Legendrian curve of $X$ is a Legendrian curve of $\widetilde{X}$.

Definition 2.1. We call the pair $(\tau: \widetilde{X} \rightarrow X, \widetilde{\mathcal{L}})$ the blow up of the logarithmic contact manifold $(X, \mathcal{L})$ along its Legendrian submanifold $\Lambda$.

THEOREM 2.2. Let $(X, \mathcal{E})$ be a quantized meromorphic contact manifold of dimension 3 , with poles along a normal crossings divisor $Y$. Let $\Lambda$ be a Lagrangian submanifold of $X$. Let $\tau: \widetilde{X} \rightarrow X$ be the blow up of $X$ along $\Lambda$.

(i) There is a meromorphic quantization $\widetilde{\mathcal{E}}$ of the logarithmic contact manifold $\widetilde{X}$ and a morphism of filtered $\mathbf{C}$-algebras

$$
\Phi: \tau^{-1} \mathcal{E} \rightarrow \widetilde{\mathcal{E}}
$$

such that $\left.\Phi\right|_{\widetilde{X} \backslash E}:\left.\left.\tau^{-1} \mathcal{E}\right|_{\widetilde{X} \backslash E} \rightarrow \widetilde{\mathcal{E}}\right|_{\widetilde{X} \backslash E}$ is an isomorphism. The morphism $\Phi$ is flat.

Given another quantization $\mathcal{E}^{\prime}$ of $\widetilde{X}$ and another morphism of filtered $\mathbf{C}$-algebras $\Phi^{\prime}$ : $\tau^{-1} \mathcal{E} \rightarrow \mathcal{E}^{\prime}$ such that $\left.\Phi^{\prime}\right|_{\widetilde{X} \backslash E}:\left.\left.\tau^{-1} \mathcal{E}\right|_{\widetilde{X} \backslash E} \rightarrow \mathcal{E}^{\prime}\right|_{\widetilde{X} \backslash E}$ is an isomorphism, there is one and only one isomorphism of filtered $\mathbf{C}$-algebras $\Psi: \widetilde{\mathcal{E}} \rightarrow \mathcal{E}^{\prime}$ such that $\Psi \Phi=\Phi^{\prime}$.

(ii) Given a holonomic $\mathcal{E}$-module $\mathcal{M}$ the $\widetilde{\mathcal{E}}$-module $\widetilde{\mathcal{M}}=\widetilde{\mathcal{E}} \otimes_{\mathcal{E}} \mathcal{M}$ is also holonomic. Moreover, the support of $\widetilde{\mathcal{M}}$ equals the proper inverse image of the support of $\mathcal{M}$.

We refer to $(2.1)$ as the blow up of the quantized contact manifold $(X, \mathcal{E})$ along $\Lambda$. We call $\widetilde{\mathcal{M}}$ the blow up of the holonomic $\mathcal{E}$-module $\mathcal{M}$ along $\Lambda$.

TheOrem 2.3. Let $M$ be a complex manifold. Let $N$ be a normal crossings divisor of $M$. Let $S$ be a smooth hypersurface of $M$, transversal to $N$. Let $\Lambda$ be the logarithmic 
conormal of $S$ relative to $N$. Let $\widetilde{X}$ be the blow up of $\mathbf{P}^{*}\langle M / N\rangle$ along $\Lambda$. Put $N_{0}=N \cup S$. Let $\Gamma$ be a Legendrian curve of $\mathbf{P}^{*}\langle M / N\rangle$.

(i) There is an open subset $W$ of $\widetilde{X}$ such that $W$ contains the proper inverse image of $\Gamma$ and there is a canonical open imersion $W \hookrightarrow \mathbf{P}^{*}\left\langle M / N_{0}\right\rangle$ such that the diagram below commutes.

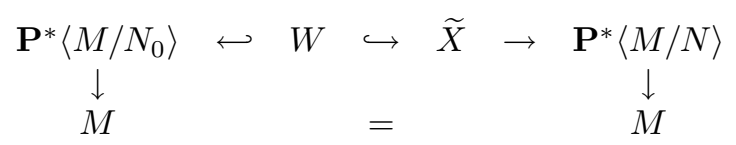

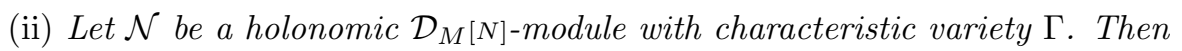

$$
\left.\left.\left(\mathcal{E}_{M\left[N_{0}\right]} \otimes_{\mathcal{D}_{M}\left[N_{0}\right]} \tilde{\mathcal{N}}\right)\right|_{W} \stackrel{\sim}{\longrightarrow}\left(\mathcal{E}_{M[N]} \otimes_{\mathcal{D}_{M}[N]} \mathcal{N}\right)^{\sim}\right|_{W}
$$

Statement 2.3(ii) says that the blow up of the microlocalization of a $\mathcal{D}_{M}[N]$-module $\mathcal{N}$ along the conormal bundle of a curve $H$ transversal to $N$ is essentially the microlocalization of the localization of $\mathcal{N}$ along $N \cup H$.

The results of geometric nature stated in this section were proved in [N2]. In order to prove the sheaf theoretic results it is convenient to sketch the proofs of the geometrical results.

Let $X=[d y-p d x / x]$ be an affine logarithmic contact manifold. Some straightforward computations show that the blow up of $X$ along the Legendrian submanifold $\{y=p=0\}$ equals the obvious patching of the affine logarithmic contact manifolds

$$
X_{y}=\left[\frac{d y}{y}-\frac{p}{y} \frac{d x}{x}\right] \quad \text { and } \quad X_{p}=\left[\frac{d x e^{-y / p}}{x e^{-y / p}}-\frac{y}{p} \frac{d p}{p}\right] .
$$

Statement 2.1(i) follows from the remark above and from Theorem 2.3(iii).

From a set theoretical point of view $E \cap\left(X_{p} \backslash X_{y}\right)$ equals $\left\{(x, y, p / y) \in X_{y}: x=\right.$ $p / y=0\}$. Hence $E \cap\left(X_{p} \backslash X_{y}\right)$ equals the intersection of $X_{p}$ with the residual set of $\tilde{X}$ along $\{x=0\}$. Statement 2.3(i) follows from the remarks above and Theorem 2.3(ii).

Let $M, M_{0}, M_{1}$ be copies of $\mathbf{C}^{2}$ with coordinates $(x, y),\left(x_{0}, y_{0}\right),\left(x_{1}, y_{1}\right)$. Put $N=$ $\{x=0\}, N_{0}=\left\{x_{0} y_{0}=0\right\}, N_{1}=\left\{x_{1} y_{1}=0\right\}$. We can look at $\mathbf{P}^{*}\langle M / N\rangle$ as the patching of the affine logarithmic contact manifolds $X$ and $\left[\frac{d x}{x}-q d y\right]$. We can look at $\mathbf{P}^{*}\left\langle M_{k} / N_{k}\right\rangle$, $k=0,1$, as the patching of the affine logarithmic contact manifolds

$$
X_{k}=\left[\frac{d y_{k}}{y_{k}}-p_{k} \frac{d x_{k}}{x_{k}}\right] \text { and }\left[\frac{d x_{k}}{x_{k}}-q_{k} \frac{d y_{k}}{y_{k}}\right] .
$$

We will identify the affine logarithmic contact manifolds $X_{y}$ and $X_{0}, X_{p}$ and $X_{1}$. Put

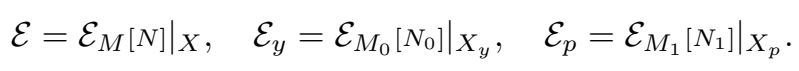

Let $P$ denote the section

$$
\sum_{k=0}^{+\infty} \frac{1}{k !} \delta_{y_{1}}^{-k} \prod_{j=0}^{k-1}\left(\delta_{x_{1}}-j\right)
$$

of the sheaf $\mathcal{E}_{p}$. We will glue the sheaves $\mathcal{E}_{y}$ and $\mathcal{E}_{p}$ by the only quantized contact transformation $\beta$ ta such that

$$
\beta\left(x_{0}\right)=y_{1} P, \quad \beta\left(y_{0}\right)=-\delta_{x_{1}} x_{1} \delta_{y_{1}}^{-1}, \quad \beta\left(\delta_{x_{0}}\right)=\delta_{y_{1}}, \quad \beta\left(\delta_{y_{0}}\right)=\delta_{x_{1}} .
$$


We obtain in this way a quantization $\widetilde{\mathcal{E}}$ of the blow up of the affine logarithmic contact manifold $X$ along $\Lambda$. We can define morphisms

$$
\Phi_{y}:\left.\tau^{-1} \mathcal{E}\right|_{X_{y}} \rightarrow \mathcal{E}_{y}, \quad \Phi_{p}:\left.\tau^{-1} \mathcal{E}\right|_{X_{p}} \rightarrow \mathcal{E}_{p}
$$

by

$$
\begin{gathered}
\Phi_{y}(x)=x_{0}, \quad \Phi_{y}(y)=y_{0}, \quad \Phi_{y}\left(\delta_{x}\right)=\delta_{x_{0}}, \quad \Phi_{y}\left(\partial_{y}\right)=\frac{1}{y_{0}} \delta_{y_{0}}, \\
\Phi_{p}(x)=y_{1} P, \quad \Phi_{p}(y)=-\delta_{x_{1}} x_{1} \delta_{y_{1}}^{-1}, \quad \Phi_{p}\left(\delta_{x}\right)=\delta_{y_{1}}, \quad \Phi_{p}\left(\partial_{y}\right)=-\frac{1}{x_{1}} \delta_{y_{1}} .
\end{gathered}
$$

We can glue these morphisms into a morphism $\Phi: \tau^{-1} \mathcal{E} \rightarrow \tilde{\mathcal{E}}$.

Given another quantization $\mathcal{E}^{\prime}$ of $\widetilde{X}$ and another morphism of filtered $\mathbf{C}$-algebras $\Phi^{\prime}: \tau^{-1} \mathcal{E} \rightarrow \mathcal{E}^{\prime}$ such that $\left.\Phi^{\prime}\right|_{\widetilde{X} \backslash E}:\left.\left.\tau^{-1} \mathcal{E}\right|_{\widetilde{X} \backslash E} \rightarrow \mathcal{E}^{\prime}\right|_{\widetilde{X} \backslash E}$ is an isomorphism, there is a quantized contact transformation $\Psi_{0}:\left.\left.\widetilde{\mathcal{E}}\right|_{\widetilde{X} \backslash E} \rightarrow \mathcal{E}^{\prime}\right|_{\widetilde{X} \backslash E}$ such that $\left.\Psi \Phi\right|_{\widetilde{X} \backslash E}=\left.\Phi^{\prime}\right|_{\widetilde{X} \backslash E}$. Let $\Psi_{y}$ be the only quantized contact transformation from $\left.\widetilde{\mathcal{E}}\right|_{X_{y}}$ to $\left.\mathcal{E}^{\prime}\right|_{X_{y}}$ such that

$$
\begin{aligned}
\Psi_{y}\left(x_{0}\right) & =\left.\Phi^{\prime}(x)\right|_{X_{y}}, & \Psi_{y}\left(y_{0}\right) & =\left.\Phi^{\prime}(y)\right|_{X_{y}}, \\
\Psi_{y}\left(\delta_{x_{0}}\right) & =\left.\Phi^{\prime}\left(\delta_{x}\right)\right|_{X_{y}}, & \Psi_{y}\left(\delta_{y_{0}}\right) & =\left.\Phi^{\prime}\left(y \partial_{y}\right)\right|_{X_{y}} .
\end{aligned}
$$

We can glue the quantized contact transformations $\Psi^{\prime}, \Psi_{y}$ into a quantized contact transformation $\Phi$ defined outside a complex submanifold of dimension 2 of $\tilde{X}$ such that $\Psi \Phi=\Phi^{\prime}$. It follows from Hartogs' Theorem that we can extend $\Phi$ to $\tilde{X}$.

4.3. Let $(X, \mathcal{L})$ be a logarithmic contact manifold of dimension 3 , with poles along a normal crossings divisor $Y$. Let $Z$ be the singular part of $Y$. Let $\tau: \widetilde{X} \rightarrow X$ be the blow up of $X$ along $Z$. Let $E$ be the exceptional divisor of $\tau$. Put $Y^{\tau}=\tau^{-1}(Y)$.

Proposition 3.1. (i) The $\mathcal{O}_{\widetilde{X}}$-module $\widetilde{\mathcal{L}}=\tau^{*} \mathcal{L}$ is a structure of logarithmic contact manifold with poles along $Y_{0}$. Moreover, $\tau$ is a contact transformation.

(ii) The proper inverse image of a Legendrian curve of $X$ is a Legendrian curve of $\tilde{X}$.

Definition 3.1. We call the pair $(\tau: \widetilde{X} \rightarrow X, \widetilde{\mathcal{L}})$ the blow up of the logarithmic contact manifold $(X, \mathcal{L})$ along the singular part of its set of poles.

THEOREM 3.2. (i) Let $(X, \mathcal{E})$ be a quantized meromorphic contact manifold of dimension 3. Let $\tau: \widetilde{X} \rightarrow X$ be the blow up of $X$ along the singular part of its set of poles. Let $E$ be the exceptional divisor of $\tau$. Then there is a meromorphic quantization $\widetilde{\mathcal{E}}$ of the logarithmic contact manifold $\widetilde{X}$ and a morphism of filtered $\mathbf{C}$-algebras

$$
\Phi: \tau^{-1} \mathcal{E} \rightarrow \widetilde{\mathcal{E}}
$$

such that $\left.\Phi\right|_{\widetilde{X} \backslash E}:\left.\left.\tau^{-1} \mathcal{E}\right|_{\widetilde{X} \backslash E} \rightarrow \widetilde{\mathcal{E}}\right|_{\widetilde{X} \backslash E}$ is an isomorphism. The morphism $\Phi$ is flat.

Given another quantization $\mathcal{E}^{\prime}$ of $\widetilde{X}$ and another morphism of filtered $\mathbf{C}$-algebras $\Phi^{\prime}$ : $\tau^{-1} \mathcal{E} \rightarrow \mathcal{E}^{\prime}$ such that $\left.\Phi^{\prime}\right|_{\widetilde{X} \backslash E}:\left.\left.\tau^{-1} \mathcal{E}\right|_{\widetilde{X} \backslash E} \rightarrow \mathcal{E}^{\prime}\right|_{\widetilde{X} \backslash E}$ is an isomorphism, there is one and only one isomorphism of filtered $\mathbf{C}$-algebras $\Psi: \widetilde{\mathcal{E}} \rightarrow \mathcal{E}^{\prime}$ such that $\Psi \Phi=\Phi^{\prime}$.

(ii) Given a holonomic $\mathcal{E}$-module $\mathcal{M}$ the $\widetilde{\mathcal{E}}$-module $\widetilde{\mathcal{M}}=\widetilde{\mathcal{E}} \otimes_{\mathcal{E}} \mathcal{M}$ is also holonomic. Moreover, the support of $\widetilde{\mathcal{M}}$ equals the proper inverse image of the support of $\mathcal{M}$. 
We refer to $(3.1)$ as the blow up of the quantized contact manifold $(X, \mathcal{E})$ along $\Lambda$. We call $\widetilde{\mathcal{M}}$ the blow up of the holonomic $\mathcal{E}$-module $\mathcal{M}$ along $\Lambda$.

Theorem 3.3. Let $M$ be a complex manifold of dimension 2. Let $N$ be a normal crossings divisor of $M$. Let $C$ be the singular part of $N$. Let $\sigma: \widetilde{M} \rightarrow M$ be the blow up of $M$ along $C$. Let $E$ be the exceptional divisor of $\sigma$. Put $N_{\sigma g m a}=\sigma^{-1}(N)$.

(i) The blow up of the contact manifold $\mathbf{P}^{*}\langle M / N\rangle$ along the singular part of its set of poles equals the logarithmic contact manifold $\mathbf{P}^{*}\left\langle\widetilde{M} / N^{\sigma}\right\rangle$. Moreover, the diagram below commutes.

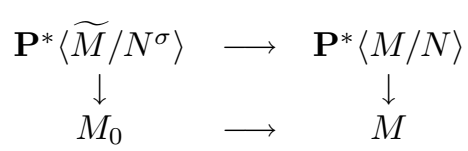

(ii) Let $\mathcal{N}$ be a holonomic $\mathcal{D}_{M}[N]$-module. Then

$$
\mathcal{E}_{M\left[N^{\sigma}\right]} \otimes_{\mathcal{D}_{M}\left[N^{\sigma}\right]} \tilde{\mathcal{N}} \stackrel{\sim}{\longrightarrow}\left(\mathcal{E}_{M}[N] \otimes_{\mathcal{D}_{M}[N]} \mathcal{N}\right)^{\sim} .
$$

Statement 3.3(ii) says that the blow up of the microlocalizaton of the $\mathcal{D}_{M[N] \text {-module }}$ $\mathcal{N}$ along the inverse image of the singular point $\left\{x^{0}\right\}$ of $N$ equals the microlocalization of the blow up of $\mathcal{N}$ along $x^{0}$.

Proposition 3.1 and statement 3.3(i) were proved in [N2]. The proofs of Theorem 3.2 and statement 3.3(ii) are similar to the proofs of similar results in the previous subsections and hence omitted.

\section{A desingularization theorem}

THEOREM 1. Let $(X, \mathcal{E})$ be a quantized contact manifold of dimension 3 . Let $\mathcal{M}$ be a holonomic $\mathcal{E}$-module. Let $\Gamma$ be the support of $\mathcal{M}$. Then there are an open subset $X_{0}$ of $X$ that contains $\Gamma$, a quantized meromorphic contact manifold $(\widetilde{X}, \widetilde{\mathcal{E}})$ with poles along a normal crossings divisor $E$, a holonomic $\widetilde{\mathcal{E}}$-module $\widetilde{\mathcal{M}}$ and a proper map $\pi: \widetilde{X} \rightarrow X_{0}$, such that:

(i) The support $\widetilde{\Gamma}$ of $\widetilde{\mathcal{M}}$ is a smooth Legendrian curve that intersects $E$ transversally.

(ii) The holomorphic map $\left.\pi\right|_{\widetilde{X} \backslash E}: \widetilde{X} \backslash E \rightarrow X$ is a contact transformation, $\pi(\widetilde{X} \backslash E)$ is an open neighbourhood of $\Gamma_{\text {reg }}$ and $\pi(\rightarrow \widetilde{\Gamma})$ equals $\Gamma$.

(iii) The restrictions of $\mathcal{M}$ and $\widetilde{\mathcal{M}}$ to $\pi(\widetilde{X} \backslash E)$ are isomorphic as systems of microdifferential equations.

Let $(X, \mathcal{L})$ be a contact manifold of dimension 3. Let $\Gamma$ be a Legendrian curve of $X$. Let us consider the following desingularization game: to blow up the contact manifold $(X, \mathcal{L})$ along centers of the three types considered in Section 4 , taking at each step the proper inverse image of the Legendrian curve $\Gamma$, in such a way that, after a finite number of explosions, the proper inverse image of $\Gamma$ is smooth and transversal to the normal crossings divisor created by the explosions.

We showed in [N2] that we can always win this game, proving theorem 2 . Theorem 1 follows from theorem 2 and statements $4 . k .2(\mathrm{ii}), 1 \leq k \leq 3$, that show that the support of the blow up of a system of meromorphic microdifferential equations only depends on the support of the system. 
TheOREM 2. Let $(X, \mathcal{L})$ be a logarithmic contact manifold of dimension 3 . Let $\Gamma$ be a Legendrian curve. Then there are an open subset $X_{0}$ of $X$ that contains $\Gamma$, a logarithmic contact manifold $(\widetilde{X}, \widetilde{\mathcal{L}})$ with poles along a normal crossings divisor $E$, a smooth Legendrian curve $\widetilde{\Gamma}$ and a proper map $\pi: \widetilde{X} \rightarrow X_{0}$, such that:

(i) The curve $\widetilde{\Gamma}$ intersects $E$ transversally.

(ii) The holomorphic map $\left.\pi\right|_{\widetilde{X} \backslash E}: \widetilde{X} \backslash E \rightarrow X$ is a contact transformation, $\pi(\widetilde{X} \backslash E)$ is an open neighbourhood of $\Gamma_{\mathrm{reg}}$ and $\pi(\Gamma)$ equals $\Gamma$.

\section{References}

[B] E. Brieskorn and H. Knörrer, Plane Algebraic Curves, Birkhäuser, Boston, 1986.

[D] P. Deligne, Equations différentielles à points singuliers réguliers, Lecture Notes in Math. 163, Springer, Berlin, 1973.

[H] L. Hörmander, The Analysis of Linear Partial Differential Operators III, Springer, Berlin, 1985.

[N1] O. Neto, Blow up for a holonomic system, Publ. Res. Inst. Math. Sci. 29 (1993), $167-233$.

[N2] - A desingularization theorem for Legendrian curves, in: Singularity Theory, D.T. Lê, K. Saito and B. Teissier (eds.), World Sci., 1995, 527-548.

[N3] - On the structure of regular holonomic systems with irreducible support, in preparation.

[SKK] M. Sato, T. Kawai and M. Kashiwara, Microfunctions and Pseudo-differential Equations, in: Hyperfunctions and Partial Differential Equations, Proc. Conf. Katata 1971, H. Komatsu (ed.), Lecture Notes in Math. 287, Springer, Berlin, 1973, 265-529.

[S] P. Schapira, Microdifferential Systems in the Complex Domain, Springer, Berlin, 1985. 